\title{
An Implicit Fourth Order Difference Method for Viscous Flows*
}

\author{
By Daniel S. Watanabe and J. Richard Flood
}

\begin{abstract}
A new implicit finite-difference scheme for viscous flows is presented. The scheme is based on Simpson's rule and two-point Hermite interpolation, is uniformly accurate to fourth order in time and space, and is unconditionally stable according to a Fourier stability analysis. Numerical solutions of Burger's equation are presented to illustrate the order and accuracy of the scheme.
\end{abstract}

1. Introduction. The invention of the electronic digital computer stimulated the intensive development of numerical methods for the solution of fluid flow problems. The majority of the methods first developed were explicit and of low order because their utility on the small slow early computers derived from their simplicity. This trend has continued to the present, despite the accuracy of high order schemes and the unconditional stability of implicit schemes. The new parallel and pipeline computers, however, have spurred interest in complex difference methods by making feasible the large calculations required by such schemes. Recently Rusanov [5] and Burstein and Mirin [2] have studied explicit third order schemes for hyperbolic systems, while Zwas and Abarbanel [6] have developed an explicit fourth order method for special hyperbolic systems. We present in this paper a new unconditionally stable implicit scheme for viscous flows which is uniformly accurate to fourth order in time and space.

We first describe the method and examine the local truncation error. We then present a linearized stability analysis of the scheme. Finally, we present several examples to illustrate the accuracy and stability of the method.

2. Difference Scheme. Consider the initial-value problem

$$
\begin{aligned}
u_{t} & =f\left(u, u_{x}, u_{x x}\right), \\
u(x, 0) & =g(x), \quad-\infty<x<\infty, \\
u(x+L, t) & =u(x, t) .
\end{aligned}
$$

Here, $u$ is an unknown vector function of $x$ and $t, f$ is a given nonlinear vector function of $u, u_{x}$, and $u_{x x}$, and $g$ is a given vector function of $x$. We assume the periodicity condition in lieu of boundary conditions. Rather than make specific differentiability assumptions, we shall assume that $u$ has derivatives of any order required.

Received January 11, 1973, revised April 2, 1973.

AMS (MOS) subject classifications (1970). Primary 65M05, 65M10.

Key words and phrases. Partial differential equations, initial-value problems, finite-difference schemes, unconditional stability, high order accuracy, viscous flows.

* This work was supported in part by the Joint Services Electronics Program (U. S. Army, U. S. Navy, and U. S. Air Force) under Contract DAAB-07-67-C-0199, and in part by the Office of Naval Research under Contract ONR N00014-67-A-0305-0019. 
The integration of (1) from $t$ to $t+\Delta t$ yields

$$
u(x, t+\Delta t)=u(x, t)+\int_{t}^{t+\Delta t} f\left(u, u_{x}, u_{x x}\right) d s .
$$

We can discretize this equation by approximating the integral by a quadrature formula employing nodes in the interval $[t, t+\Delta t]$. Approximations at the interior nodes to $u$, and hence $f$, can then be obtained from $u$ and $u_{t}$ at times $t$ and $t+\Delta t$ through Hermite interpolation. Since the error in Hermite interpolation is of $O\left(\Delta t^{4}\right)$, we should employ a quadrature formula with error of $O\left(\Delta t^{5}\right)$. Simpson's rule is such a formula and, moreover, employs only one interior node, thus minimizing the number of interpolations. Of course, $f$ cannot be computed exactly because it involves derivatives of $u$ with respect to $x$. However, we can approximate $f$ by replacing $u_{x}$ and $u_{x x}$ by appropriate difference quotients. If these quotients are chosen as the centered fivepoint formulae, the overall accuracy of the scheme is preserved.

We seek a mesh function $U$ which approximates the exact solution $u$ on the mesh

$$
\left\{x_{ \pm i}= \pm i \Delta x, t_{n}=n \Delta t ; i, n=0,1,2, \cdots\right\} .
$$

We shall restrict our attention to meshes where $\Delta x=\Delta t / r=\Delta$ for some positive constant $r$. Let $u_{i}{ }^{n}$ and $U_{i}{ }^{n}$ denote the values of $u$ and $U$ at the mesh point $\left(x_{i}, t_{n}\right)$, and let

$$
f_{d}(u)=f\left(u, d_{x} u, d_{x}^{2} u\right), \quad f_{D}(u)=f\left(u, D_{x} u, D_{x}^{2} u\right),
$$

where $d_{x}=\partial / \partial x, d_{x}{ }^{2}=\partial^{2} / \partial x^{2}$, and $D_{x}$ and $D_{x}{ }^{2}$ are the corresponding five-point centered difference quotients

$$
\begin{aligned}
& D_{x} u_{i}=\left(u_{i-2}-8 u_{i-1}+8 u_{i+1}-u_{i+2}\right) / 12 \Delta x, \\
& D_{x}^{2} u_{i}=\left(-u_{i-2}+16 u_{i-1}-30 u_{i}+16 u_{i+1}-u_{i+2}\right) / 12 \Delta x^{2} .
\end{aligned}
$$

The method is defined by

$$
\begin{aligned}
U_{i}^{n+1} & =U_{i}^{n}+(\Delta t / 6)\left[f_{D}\left(U_{i}^{n}\right)+4 f_{D}\left(\bar{U}_{i}^{n+1 / 2}\right)+f_{D}\left(U_{i}^{n+1}\right)\right], \\
U_{i}^{n+1 / 2} & =\left(U_{i}^{n}+U_{i}^{n+1}\right) / 2+(\Delta t / 8)\left[f_{D}\left(U_{i}^{n}\right)-f_{D}\left(U_{i}^{n+1}\right)\right], \\
& i=0,1, \cdots, I-1 ; n=0,1, \cdots,
\end{aligned}
$$

where $I=L / \Delta x$. These equations define a system of nonlinear algebraic equations which must be solved at each time step through some iterative procedure.

The local truncation error is defined by

$$
e(u)=\int_{t_{n}}^{t_{n+1}} f_{d}(u) d s-(\Delta t / 6)\left[f_{D}\left(u^{n}\right)+4 f_{D}\left(\bar{u}^{n+1 / 2}\right)+f_{D}\left(u^{n+1}\right)\right],
$$

where the spatial indices are suppressed for convenience. Our choice of $D_{x}$ and $D_{x}{ }^{2}$ implies that

$$
\begin{aligned}
& D_{x} u=d_{x} u-\Delta^{4} d_{x}^{5} u / 30+O\left(\Delta^{6}\right), \\
& D_{x}^{2} u=d_{x}^{2} u-\Delta^{4} d_{x}^{6} u / 90+O\left(\Delta^{6}\right) .
\end{aligned}
$$

Substituting this result in $f_{D}(u)$ and expanding, we find that 


$$
f_{D}(u)=f_{d}(u)-\Delta^{4} \alpha(x, u)+O\left(\Delta^{6}\right)
$$

where

$$
\alpha(x, u)=\left(\partial / \partial u_{x}\right) f_{d}(u) d_{x}^{5} u / 30+\left(\partial / \partial u_{x x}\right) f_{d}(u) d_{x}^{6} u / 90 .
$$

It follows from (2b), (4), and the two-point Hermite interpolation formula

$$
u^{n+1 / 2}=\left(u^{n}+u^{n+1}\right) / 2+(\Delta t / 8) d_{t}\left(u^{n}-u^{n+1}\right)+\Delta t^{4} d_{t}^{4} u^{n+1 / 2} / 384+O\left(\Delta t^{6}\right)
$$

that the interpolate $\bar{u}^{n+1 / 2}$ satisfies

$$
\bar{u}^{n+1 / 2}=u^{n+1 / 2}-\Delta^{4} \beta(x, u)+O\left(\Delta^{6}\right),
$$

where

$$
\beta(x, u)=r^{4} d_{t}^{4} u^{n+1 / 2} / 384+(r \Delta / 8)\left[\alpha\left(x, u^{n}\right)-\alpha\left(x, u^{n+1}\right)\right]
$$

Clearly,

$$
\begin{aligned}
& D_{x} \bar{u}^{n+1 / 2}=d_{x} u^{n+1 / 2}-\Delta^{4} d_{x} \beta(x, u)+O\left(\Delta^{4}\right), \\
& D_{x}^{2} \bar{u}^{n+1 / 2}=d_{x}^{2} u^{n+1 / 2}-\Delta^{4} d_{x}^{2} \beta(x, u)+O\left(\Delta^{4}\right),
\end{aligned}
$$

and hence as before

$$
f_{D}\left(\bar{u}^{n+1 / 2}\right)=f_{d}\left(u^{n+1 / 2}\right)+O\left(\Delta^{4}\right) .
$$

Since the error in Simpson's rule is of $O\left(\Delta^{5}\right)$, it follows from (3), (4), and (5) that $e(u)=O\left(\Delta^{5}\right)$, and the scheme is uniformly accurate to fourth order in time and space.

3. Stability. We shall examine the stability of the scheme (2) by applying it to the system of linear partial differential equations

$$
u_{t}=A u_{x x}+B u_{x},
$$

where $A$ and $B$ are constant matrices such that the matrix $C$, defined by

$$
C=\alpha A+i \Delta x \beta B
$$

with $\alpha=(-15+16 \cos \phi-\cos 2 \phi) / 6, \beta=(8 \sin \phi-\sin 2 \phi) / 6$, has eigenvalues $c=\lambda+i \mu$, satisfying $\lambda \leqq 0$ for $0 \leqq \phi<2 \pi$. This assumption is satisfied, for example, by the linearized Navier-Stokes equations for which $u=[\rho, v, p]^{T}$ and

$$
A=\left[\begin{array}{ccc}
0 & 0 & 0 \\
0 & 4 /(3 \operatorname{Re} \rho) & 0 \\
-\gamma p /\left(\operatorname{Re} \operatorname{Pr} \rho^{2}\right) & 0 & \gamma /(\operatorname{Re} \operatorname{Pr} \rho)
\end{array}\right], \quad B=\left[\begin{array}{ccc}
-v & -\rho & 0 \\
0 & -v & -1 / \rho \\
0 & -\gamma p & -v
\end{array}\right] \text {, }
$$

where $\rho, v$, and $p$ are the density, velocity, and pressure per unit volume, $\operatorname{Re}$ and $\operatorname{Pr}$ are the Reynolds and Prandtl numbers, and $\gamma$ is the ratio of specific heats. For the case of viscosity without heat conduction where $\operatorname{Pr}=\infty$, the eigenvalues of $C$ are easily determined and are

$$
\begin{gathered}
c_{1}=-i \Delta x \beta v \\
c_{2,3}=2 \alpha /(3 \mathrm{Re} \rho)-i \Delta x \beta v \pm\left[4 \alpha^{2} /\left(9 \mathrm{Re}^{2} \rho^{2}\right)-\beta^{2} \Delta x^{2} \gamma p / \rho\right]^{1 / 2} .
\end{gathered}
$$


Since $\alpha \leqq 0$ and $\rho, p \geqq 0$, the assumption is clearly satisfied. For the more realistic case where $\operatorname{Pr}=1$, we chose not to calculate the eigenvalues but to demonstrate using the Routh-Hurwitz conditions that the eigenvalues have nonpositive real parts. The calculation is straightforward but extremely tedious and hence is omitted.

We follow von Neumann and assume a perturbation of the form $u^{n}=\xi^{n} \exp (i \omega x)$. Setting $f_{D}(u)=A D_{x}^{2} u+B D_{x} u$ and substituting the perturbation in (2), we find that the amplitude of the perturbation satisfies

$$
\xi^{n+1}=G \xi^{n},
$$

where the amplification matrix $G$ has the form

$$
G=\left(I-\epsilon C / 2+\epsilon^{2} C^{2} / 12\right)^{-1}\left(I+\epsilon C / 2+\epsilon^{2} C^{2} / 12\right)
$$

with $\phi=\omega \Delta x$ and $\epsilon=\Delta t / \Delta x^{2}$. A simple calculation shows that the eigenvalues $g$ of $G$ satisfy

$$
|g|^{2}=\left[\nu+\lambda \epsilon+\lambda\left(\lambda^{2}+\mu^{2}\right) \epsilon^{3} / 12\right] /\left[\nu-\lambda \epsilon-\lambda\left(\lambda^{2}+\mu^{2}\right) \epsilon^{3} / 12\right],
$$

where $\nu>0$. Since $\lambda \leqq 0$ by hypothesis, it follows that $|g| \leqq 1$ for any $\Delta t$ and $\Delta x$, and the method is unconditionally stable.

The system of nonlinear equations (2) must be solved carefully to maintain the stability of the method. We have solved this system using successive substitution, nonlinear overrelaxation, and Broyden's quasi-Newton method [1]. Unfortunately, the first two methods often converged only for $\Delta t=O\left(\Delta x^{2}\right)$, nullifying the main advantage of the scheme. Broyden's method, however, worked well, always allowing us to take $\Delta t=O(\Delta x)$.

Our discussion of the order and stability of the method is valid only for problems with periodic boundary conditions. In a general initial-boundary-value problem, our scheme, like other high order schemes, cannot be applied at the nodes adjacent to the boundaries. There are several ways to handle these critical nodes. For example, we might use appropriate uncentered difference equations, or employ extrapolation to generate any values required by the centered equations at fictitious nodes outside the boundaries. It is easy to invent methods based on these ideas, but they often place undesirable restrictions on the time step. This important problem merits further study.

4. Numerical Examples. We have tested our scheme on a variety of problems and present here selected results to illustrate the accuracy and stability of the method.

Burger's equation is often used as a model for the one-dimensional time-dependent Navier-Stokes equations. It approximates to first order the motion of a plane wave of small but finite amplitude. The equation takes account of convection and diffusion, and has the form

$$
u_{t}+\left(u^{2} / 2\right)_{x}=\lambda u_{x x},
$$

where $u$ is the excess wavelet velocity, and $\lambda$ is the diffusivity of sound.

We studied Burger's equation subject to the initial condition

$$
u(x, 0)=\sin \pi x, \quad-\infty<x<\infty .
$$

This problem has the exact solution 


$$
u(x, t)=-2 \lambda \phi_{x}(x, t) / \phi(x, t)
$$

where

$$
e^{\mu} \phi(x, t)=I_{0}(\mu)+2 \sum_{n=1}^{\infty} I_{n}(\mu) \exp \left(-\lambda n^{2} \pi^{2} t\right) \cos n \pi x,
$$

$\mu=1 /(2 \pi \lambda)$, and $I_{n}$ is the modified Bessel function of order $n$. The solution has a period $L$ of 2 and is antisymmetric with respect to the lines $x=0, \pm 1, \pm 2, \cdots$. Hence, we need only consider the interval $0 \leqq x \leqq 1$. We computed the solution of this problem on the region $0 \leqq x \leqq 1,0 \leqq t \leqq 1$ for $\lambda=.05$, and $\Delta x=\Delta t=.05$, .025 , and .0125 .

We also studied Burger's equation subject to the initial condition

$$
u(x, 0)=2 \operatorname{ch}(-x), \quad-\infty<x<\infty,
$$

where $h$ is Heaviside's step function. The solution is a shock-like wave moving with velocity $c$. For computational convenience, we transformed the space coordinate into a coordinate moving with velocity $c$. In this new coordinate, the solution is

$$
u(x, t)=2 c /[1+\exp (c x / \lambda) \operatorname{erfc}[-(x+c t) /(2 \sqrt{ }(\lambda t))] / \operatorname{erfc}[(x-c t) /(2 \sqrt{ }(\lambda t))]] .
$$

We computed the solution of the transformed problem on the region $-1 \leqq x \leqq 1$, $.05 \leqq t \leqq 3.05$ for $\lambda=.1, c=.5$, and $\Delta t=\Delta x=.2, .1$, and .05 . The initial and boundary values, including those at the fictitious nodes outside the boundaries, were obtained from the exact solution. The calculation was started at $t=.05$, rather than $t=0$, to preserve the order of the scheme. It should be noted, however, that the method produces smooth solutions when started with the step function.

Table 1 lists the error norms and computed orders

\begin{tabular}{|c|c|c|c|c|c|c|c|c|}
\hline problem & time & $\Delta$ & $\|e\|_{1}$ & $\|e\|_{2}$ & $\|e\|_{\infty}$ & $r_{1}$ & $r_{2}$ & $r_{\infty}$ \\
\hline \multirow[t]{5}{*}{1} & 1.00 & .0500 & $7.77-5$ & $1.52-4$ & $5.06-4$ & & & \\
\hline & & & & & & 3.9 & 3.9 & 3.8 \\
\hline & & .0250 & $5.19-6$ & $1.04-5$ & $3.65-5$ & & & \\
\hline & & & & & & 4.0 & 4.0 & 3.9 \\
\hline & & .0125 & $3.31-7$ & $6.67-7$ & $2.37-6$ & & & \\
\hline \multirow[t]{5}{*}{2} & 3.05 & .2000 & $6.88-4$ & $9.41-4$ & $1.81-3$ & & & \\
\hline & & & & & & 3.7 & 3.8 & 3.8 \\
\hline & & .1000 & $5.31-5$ & $6.98-5$ & $1.34-4$ & & & \\
\hline & & & & & & 4.0 & 3.9 & 3.7 \\
\hline & & .0500 & $3.43-6$ & $4.58-6$ & $1.03-5$ & & & \\
\hline
\end{tabular}

$$
\begin{gathered}
\|e\|_{a}=\left(\sum_{i}\left|e_{i}\right|^{a} / m\right)^{1 / a}, \\
r_{a}(\Delta)=\ln \left(\|e(\Delta)\|_{a} /\|e(\Delta / 2)\|_{a}\right) / \ln (2),
\end{gathered}
$$

where $e_{i}=u_{i}-U_{i}$, and the sum is taken over the $m$ interior nodes. The computed

TABLE 1. Error norms and computed orders 
TABLE 2. Comparison of explicit and implicit methods

\begin{tabular}{llccr}
\hline \multicolumn{1}{c}{ method } & $\Delta x$ & $\Delta t$ & error & time \\
\hline present & .1000 & .02500 & $3.5-4$ & 1.0 \\
Rubin and Burstein & .0100 & .00005 & $3.4-4$ & 190.3 \\
present & $.0909 \ldots$ & .02000 & $2.0-4$ & 1.4 \\
MacCormack & .0100 & .00005 & $1.8-4$ & 149.7 \\
\hline
\end{tabular}

orders should be 4 in both problems since the centered formulae are applied at each node. The scheme clearly fulfills our expectations.

We tested the stability of the scheme by solving the first problem with $\Delta t \gg \Delta x$. As expected, it worked extremely well. For example, for $\lambda=.1$ we used $\Delta t / \Delta x=$ $.6 / .01=60$ without any difficulty. In each case, the maximum allowable time step appeared to be governed only by the allowable discretization error.

The maximum allowable time step for explicit schemes is generally directly proportional to $\Delta x^{2}$ and inversely proportional to the viscosity. This restriction, coupled with the fine spatial meshes required by low order methods to achieve high accuracy, makes explicit low order schemes inefficient for highly viscous flows. Our scheme, however, is well suited to such problems. To illustrate this fact, we computed the solution of the first problem on the region $0 \leqq x \leqq 1,0 \leqq t \leqq .2$ for $\lambda=1$ using MacCormack's [3] and Rubin and Burnstein's [4] explicit second order methods and our scheme. The largest time step consistent with stability was used for each explicit method. The solutions are compared in Table 2. The errors listed are maximum relative errors and the time unit is equivalent to .2 seconds on an IBM $360 / 75$.

Department of Computer Science and

Coordinated Science Laboratory

University of Illinois

Urbana, Illinois 61801

1. C. G. Broyden, "A new method of solving nonlinear simultaneous equations," Comput. J., v. 12, 1969/70, pp. 94-99. MR 39 \#6509.

2. S. Z. Burstein \& A. A. Mirin, "Third order difference methods for hyperbolic equations," J. Computational Phys., v. 5, 1970, pp. 547-571. MR 43 \#8255.

3. R. W. MACCORMACK, "Numerical solution of the interaction of a shock wave with a laminar boundary layer," Lecture Notes in Physics, v. 8, Springer-Verlag, Berlin, 1971, pp. 151-163.

4. E. L. Rubin \& S. Z. Burstein, "Difference methods for the inviscid and viscous equations of a compressible gas," J. Computational Phys., v. 2, 1967, pp. 178-196.

5. V. V. RuSANOV, "On difference schemes of third order accuracy for nonlinear hyperbolic systems,” J. Computational Phys., v. 5, 1970, pp. 507-516. MR 43 \#1452.

6. G. ZWAS \& S. ABARBANEL, "Third and fourth order accurate schemes for hyperbolic equations of conservation law form," Math. Comp., v. 25, 1971, pp. 229-236. 